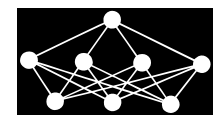

\title{
ON THE CELLULAR MECHANISMS UNDERLYING WORKING MEMORY CAPACITY IN HUMANS
}

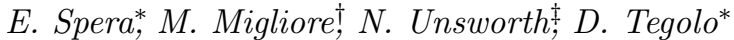

\begin{abstract}
The cellular processes underlying individual differences in the Working Memory Capacity (WMC) of humans are essentially unknown. Psychological experiments suggest that subjects with lower working memory capacity (LWMC), with respect to subjects with higher capacity (HWMC), take more time to recall items from a list because they search through a larger set of items and are much more susceptible to interference during retrieval. However, a more precise link between psychological experiments and cellular properties is lacking and very difficult to investigate experimentally. In this paper, we investigate the possible underlying mechanisms at the single neuron level by using a computational model of hippocampal CA1 pyramidal neurons, which have been suggested to be deeply involved in the recognition of specific items. The model makes a few experimentally testable predictions on the cellular processes underlying the cumulative latency in delayed free recall experimentally observed in humans under different testing conditions. The results suggest, for the first time, a physiologically plausible explanation for individual performances, and establish a proof of principle for the hypothesis that HWMC individuals use a larger portion of the apical tree with a correlated higher level of synaptic background noise.
\end{abstract}

Key words: working memory capacity, CA1, hippocampus, data analysis

Received: May 10, 2015

DOI: $10.14311 / \mathrm{NNW} .2016 .26 .019$

Revised and accepted: July 8, 2016

\section{Introduction}

Several experimental studies have convincingly demonstrated that individual differences in the Working Memory Capacity (WMC) of humans is related to differences in many abilities $[7,11,32,34]$, especially those related to the processes underlying the retrieval of information under conditions of interference $[19,38]$, and it has been related to a number of higher brain functions [12]. Individual who score in the upper or lower quartile on a variety of working memory capacity tasks such as the reading span and operation span tasks are usually classified as high (HWMC)

\footnotetext{
*Emiliano Spera, Domenico Tegolo - Corresponding author, Dipartimento di Matematica e Informatica, Universitá degli studi di Palermo, Palermo, Italy, E-mail: domenico.tegolo@unipa.it

${ }^{\dagger}$ Michele Migliore, Institute of Biophysics, National Research Council, Palermo, Italy

${ }^{\ddagger}$ Nash Unsworth, Department of Psychology, University of Oregon, Eugene, OR 97403, USA
} 
or low (LWMC) working memory capacity subjects, respectively. It has been suggested that HWMC subjects are better at suppressing thoughts about a designated event, whereas LWMC individuals are less capable to effectively suppress irrelevant thoughts [4].

Working memory capacity is often measured by analyzing the temporal dynamics of (immediate or delayed) free recall of items from a list. Here we will consider the cumulative latency in delayed free recall. Experimental findings suggested that low-WMC individuals are much more susceptible to interference during retrieval than high-WMC individuals, and it has been argued [37] that one of the possible mechanisms affecting WMC is the search strategy followed by each individual during the retrieval process.

The hypothesis is that low-WMC individuals take more time to recall items from a list because they search through a larger set of items with respect to high-WMC individuals. However, the possible cellular mechanisms underlying this effect are still unknown, relatively unexplored, and quite difficult to test experimentally at the physiological level in humans, given the rather invasive techniques that would be required. To shed light on these mechanisms, we used a modeling approach to reproduce a set of experimental data [37].

It is well known that the processes related to short-term memory encoding and retrieval involve the temporal lobe and, in particular, the hippocampal region $[1,28,33]$. In general, the expression of a higher brain function at a behavioral level is the end result of a number of independent processes occurring at different levels, from brain regions interaction, to network and circuit connectivity, to subcellular biochemical pathways. At each level there can be mechanisms that are crucial for the emergence of a specific higher brain function. In this paper we were interested in exploring the processes occurring at the single cell level that may contribute to the working memory capacity observed at the behavioral level. From this point of view, the pyramidal neurons in the CA1 region are in a crucial position to participate in these processes, because they represent the main output stage of the hippocampal circuitry [18]. Their morphological and electrophysiological properties [27] suggest that they are exquisitely tailored to modulate the processes underlying the shortterm memory.

Experimentally, it has been shown that individual neurons in the medial temporal lobe are selectively activated by specific input items [5,30,31], and that pyramidal cells are highly selective, with hippocampal pyramidal cells exhibiting the highest degree of selectivity [17]. Computational models [25, 29] have suggested that the oblique dendrites of CA1 pyramidal neurons can effectively act as independent units modulating the processes of object recognition [26].

In this work we thus used a biophysical model of CA1 neurons to investigate the possible mechanisms that can affect short-term memory at the single neuron level. We found that the number of oblique dendrites representing an item, correlated with the level of background synaptic noise, can be associated to the main features observed in the experimental findings. The results suggest a physiologically plausible explanation for the observed individual differences in the cumulative latency in delayed free recall experiments between high-WMC and low-WMC subjects. 
Spera E. et al.: On the cellular mechanisms underlying working memory...

\section{Materials and methods}

All simulations were implemented using the NEURON simulation environment [15]. Most of the simulations and analyses were carried out on a parallel supercomputer system (CINECA consortium, Bologna, Italy).

For all simulations we used cell $c 91662$, originally downloaded from the public archive neuromorpho.org [2]. The set of active and passive properties were identical to those in [26]. In the model, already validated against a number of different experimental findings on electrophysiological and synaptic integration properties in CA1 neurons $[13,14,16,21,23,24]$, sodium and DR-type potassium channels were uniformly distributed throughout the dendrites, whereas $A$-type potassium and $I h$ channels were linearly increasing with distance from the soma. Model and simulation files specifically used for this work will be available for public download under the ModelDB section of the Senselab database http://senselab.med.yale.edu.

For the purpose of the paper, we considered the 26 oblique dendrites stemming out from the main trunk of our model neuron. Random groups of $n$ oblique dendrites ( $n$-tuple), with $n$ ranging from 1 to 26 , were stimulated with synaptic inputs modeling the synchronous activation of a group of synapses.

Synaptic activation on each dendrite was modeled with a double exponential conductance change ( 0.4 and $1 \mathrm{~ms}$ for raise and decay time, respectively), with a peak conductance strong enough to ensure a local AP under all conditions of synaptic background noise (80 ns).

To model dendritic background synaptic activity, we included the Destexhe et al (2001) mechanism [9] in all compartments of the main apical trunk (as in [26]); the excitatory and inhibitory conductance are described by a stochastic random-walk similar to the Ornstein-Uhlenbeck process [35], and it has been shown that it can successfully recreate the somatic background activity observed in vivo [9]. Different Root Mean Square (RMS) levels of synaptic background activity were modeled with different peak values and standard deviation of the fluctuating excitatory and inhibitory conductance. In all cases, excitation and inhibition was balanced in such a way to obtain an average somatic resting potential of $-65 \mathrm{mV}$.

To simulate a set of cumulative recall curves, we used the number $(n)$ of active dendrites encoding an item and the level of random background synaptic activity (noise) as simulation parameters. It should be noted that the number of active dendrites used to encode objects (a list of words, in the experiments discussed in this work) implies a different number of possible ways in which the activity in a group of dendrites can be integrated in such a way to generate a somatic action potential. For example, the particular CA1 pyramidal neuron morphology we used for all simulations has 26 oblique dendrites. If words are coded using 17 obliques, for example corresponding to 17 different (and in general abstract) features, this particular neuron can be involved in the encoding of up to $\left(\begin{array}{l}26 \\ 17\end{array}\right)=$ 3, 124, 550 different words. Using 22 features (and thus 22 dendrites), this number would be reduced to $\left(\begin{array}{l}26 \\ 22\end{array}\right)=14,950$. Assuming that during the recall phase stored items are continuously presented to the involved neuron, this condition would take less time to generate a somatic action potential (i.e. a recognition signal), because only 14,950 items would be presented for eventual recognition instead of $3,124,550$. 
A total of 520 simulations were carried out to test combinations of both parameters within a uniform range of 1-26 for $n$ and $0.9-1.5 \mathrm{mV}$ for the background noise as explained in Section 3. To calculate average values, each simulation was repeated 102 times, randomizing the dendritic location of the potentiated synapses and the pattern of background activity; they were then used to form $k$ different ensembles of 6-, 9-, and 12-simulations (resulting in $k=17,11$, and 8 values from which the average recall time for 6-, 9-, and 12-words list, respectively, was calculated). A cumulative recall was calculated as the number of words recalled in the time's interval $\left[0, t_{i}\right]$.

\section{Results}

To test our model we reanalyzed and used data from a set of tests previously carried out on 23 individuals with a high working memory capacity (HWMC) and 20 individuals with low working memory capacity (LWMC) [36]. Briefly, all individuals performed delayed free-recall tasks using list of words of different lengths $(6,9$, or 12 items), and the cumulative recall was recorded as a function of recall time. Typical experimental findings are plotted in Fig. 1a for HWMC individuals tested on lists of 12 items (Fig. 1a, blue) and LWMC individuals tested on lists of 6 items (Fig. 1a, red).

It has already been shown [39] that the cumulative number of words recalled as a function of time, $\mathrm{WR}(t)$, is well described by the following exponential cumulative function

$$
A=1-\mathrm{e}^{-\lambda t},
$$

where $A$ is the asymptotic number of words recalled and $\lambda$ is the rate of approach $A$. In general, the different WMC of different individuals can be represented with different values of $A$ and $\lambda$. Typical best fits and parameters' value for three individuals tested under different conditions are shown in Fig. 1b, and the complete set of parameters fitting the performances of all individuals under all conditions is plotted in Fig. 1c. As can be seen, for practically all testing conditions there is a considerable overlap among the values of $A$ and $\lambda$ for high- or low-WMC individuals. In all cases, as shown in Fig. 1d for the typical example of HWMC and LWMC individuals tested on lists of 9 words, the distribution of $A$ and $\lambda$ values obtained from the two population were not significantly different (Mann-Whitney rank-sum test $p=1.0$ and $p=0.836$, respectively).

This suggests that there is a complex interaction among the different processes used by different individuals in the recall task, which has so far precluded a deeper understanding of the underlying physiological mechanisms.

In this work we were interested to test how the working memory capacity in humans can depend, at least in part, on the synaptic integration process occurring at the single neuron level. In our model the working memory task (to signal the time of recall of a previously seen word) is assumed to be carried out by a small group of neurons $(6,9$, or 12 , according to the word list size). We will also use the simplifying assumption that individual objects (words, in this case) are encoded by single neurons (rather than a small group of them). It should be stressed that this framework is consistent with experimental findings $[17,31]$ demonstrating 
Spera E. et al.: On the cellular mechanisms underlying working memory...

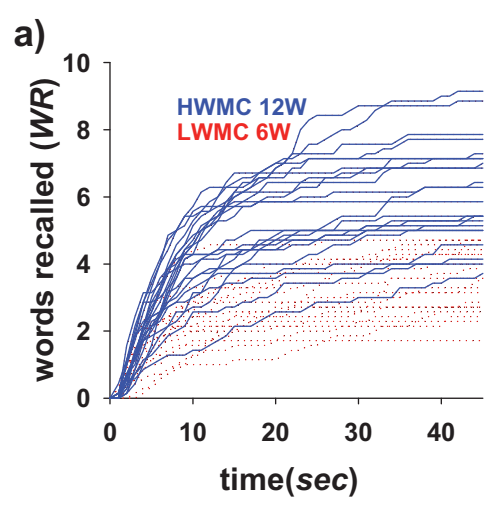

c)

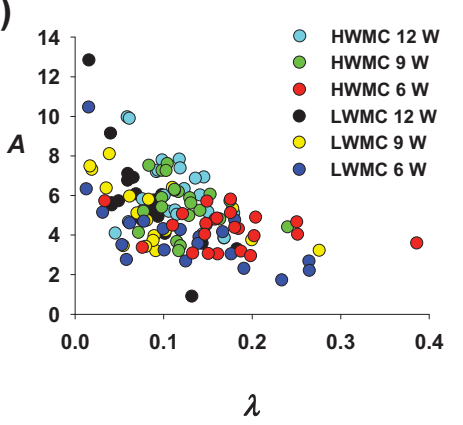

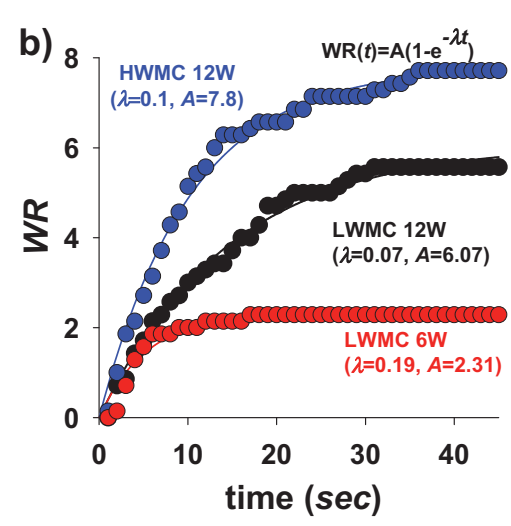

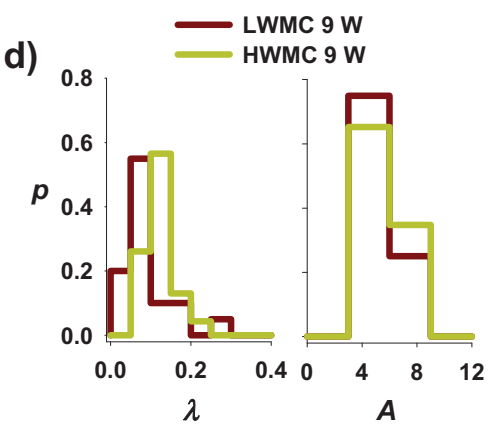

Fig. 1 Individual differences in working memory capacity. a) Typical results obtained for the number of words recalled as a function of time, in experiments with individuals with high (HWMC) or low (LWMC) working memory capacity using a search set with 12 for HWMC (blue) or 6 for LWMC items (red); b) Typical best fit of the results from 3 individuals with an exponential cumulative recall function; c) Values of fitting parameters obtained from all the experiments considered in this work. Each individual with a low or high working memory capacity (HWMC, $L W M C)$ was tested with search sets of different size $(6 \mathrm{~W}, 9 \mathrm{~W}, 12 \mathrm{~W})$; d) Distribution of $\lambda$ and $A$ for lists of 9 words.

that object representation in the hippocampus is sparse (i.e. encoded by a small number of neurons) and explicit (i.e. the presence of an object can, in principle, be reliably decoded from a very small number of neurons). Since the recall process can be expected to be modulated by the activity directly or indirectly generated by sensory signals, we decided to explore the effects of two sets of inputs that could represent them, i.e. 1) suprathreshold inputs eliciting local action potentials on the oblique dendrites, and 2) subthreshold inputs generating random background activity. The strong input on the oblique dendrites can be considered to represent information directly related to the encoding and retrieval processes, whereas the random background activity can be assumed to represent the concurrent internal cortical dynamics generated by processes related to the overall sensory inputs [8]. 
This relatively simple hypothesis cannot reveal all the possible interactions that are probably in effect during a free recall process. For example, in this work we do not expect to draw any powerful definitive inferences on the effects of cognitive loads, attention, and brain regions interaction. However, we expect to obtain very specific information on how a couple of fundamental cellular mechanisms can modulate the overall process.

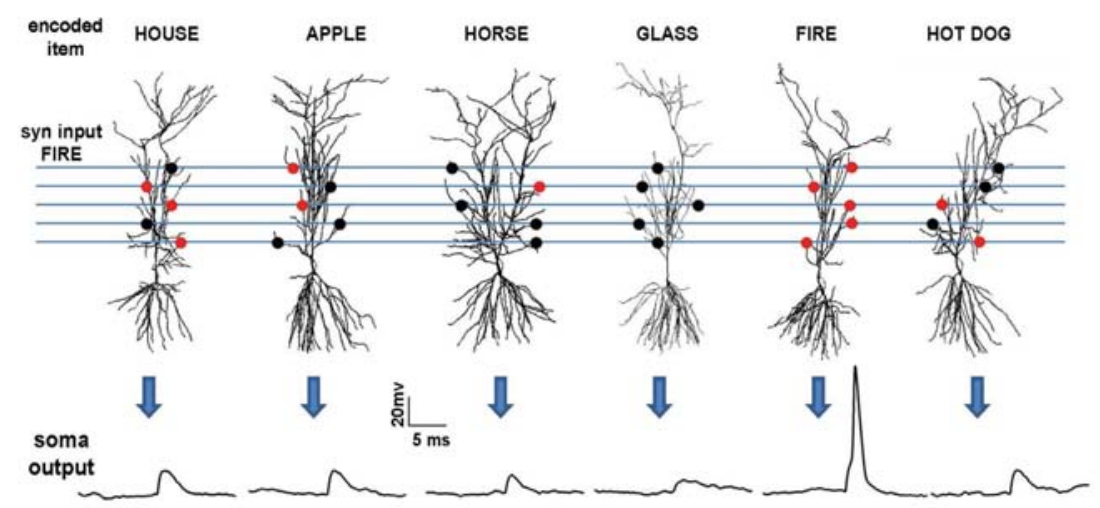

Fig. 2 Schematic representation of how different $C A 1$ neurons respond to the same input. Neurons were assumed to encode different items (top) using different combinations of $n=5$ oblique dendrites with potentiated synapses; a synaptic input may (middle, red points) or may not (middle, black points) elicit a dendritic action potential. Only when many of the $n$ inputs generate a local action potential, the overall signal will be strong enough to generate a somatic action potential (bottom).

The resulting processing is consistent with experimental evidence [20] suggesting that inputs contribute directly to dendritic spikes, with output from multiple branches summing in the axon. Following the experimental suggestion that individual branches of CA1 pyramidal neurons might be used to store recent experience [22], we assumed that each oblique dendrite can be used to detect (i.e. eliciting a local action potential that will not propagate to the soma) the presence of a specific input feature (e.g. color, shape, or other qualities), as schematically represented in Fig. 2. The learning process occurring during presentation of a list of items will thus result, through synaptic plasticity mechanisms (not explicitly implemented in this work), in a Long-Term Potentiation of synapses targeting oblique dendrites coding for specific input features. The end result will be that different neurons will be tuned to recognize (by means of a somatic action potential) the simultaneous presence of $n$ different features, as shown in Fig. 2. Activation of a strong synaptic input will generate a local (dendritic) action potential. However a somatic AP will be elicited only when an item, composed by a specific combination of $n$ features, activates enough obliques of the same neuron. As schematically represented in Fig. 2 for items encoded with $n=5$ features, in the presence of the input corresponding to "FIRE" only one neuron will have enough synaptic activity to generate a somatic action potential (i.e. to recognize the word "FIRE"). 
Spera E. et al.: On the cellular mechanisms underlying working memory...

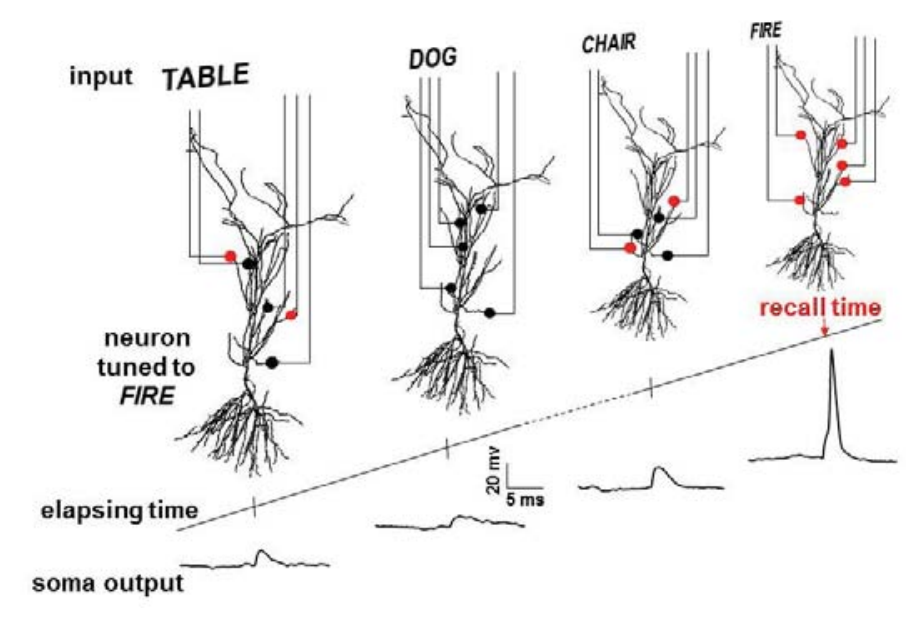

Fig. 3 Schematic representation of a neuron response as a function of time. Different synaptic inputs, representing different items (top) are sequentially activated on different dendrites of a given neuron (middle); an action potential will be generated only if and when many of the 5 potentiated synaptic inputs are activated (bottom).

The model implementation used to reproduce the recall of words from a list as a function of time is schematically illustrated in Fig. 3. During a simulation, a different random $n$-tuple of synaptic inputs (corresponding to different input words) was activated every $16 \mathrm{~ms}$ (corresponding to $\approx 60 \mathrm{~Hz}$, in the range of the $\gamma$ rhythm) in a set of 6,9 , or 12 replica of our model neuron (corresponding to the lists length used in the experiments). Each simulation lasted for $45 \mathrm{sec}$ (as in the experiments), and the somatic spike times of each neuron marked the recall time for each word.

The modeled recall curves (obtained as explained in Section 2)were compared with experiments using $\chi^{2}$ values to find the curve that best matched each experimental case. Three typical experimental cases for 12-word lists are compared with the modeling results in Fig. 4, where we show the experimental curves obtained from three individuals (Fig. 4a, black triangle), the simulation results (Fig. 4a, red circle), and the best fit of the simulation data with an exponential cumulative recall function (Fig. 4a, blue lines). Surprisingly, given the relative simple set of parameters compared with the expected complexity of the overall recall process in humans, in all cases we were able to quantitatively reproduce all experimental findings $\left(\chi^{2}\right.$ test $\left.p<0.01\right)$. The data obtained from the simulations were in very good agreement with those obtained from experiments, as shown in Fig. 4b and 4c with the set of $A$ and $\lambda$ obtained from the simulations statistically indistinguishable from those obtained experimentally (Mann-Whitney rank sum test, $p$ values are indicated in Fig. $4 \mathrm{~b}-\mathrm{c}$ ). It is important to stress that both parameters (i.e. the number of active dendrites encoding an item and the level of random background synaptic activity) have a critical role to determine a good representation of the experimental findings. As show in Fig. $4 \mathrm{~d}-\mathrm{e}$ the values of $A$ and $\lambda$ obtained from simulations using a constant noise level $(1.35 \mathrm{mV}$, corresponding to the value with the best overall $\chi^{2}$ ) gave a significantly worse reproduction of the experimental 


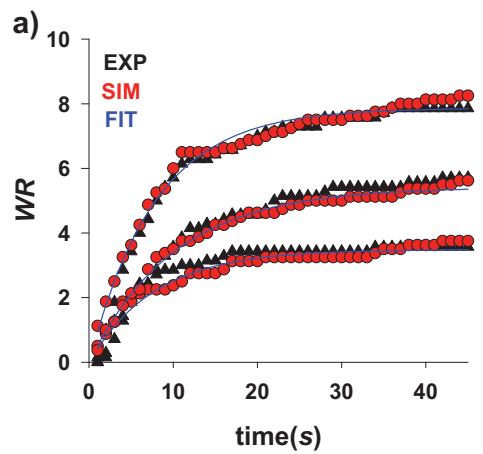

b)

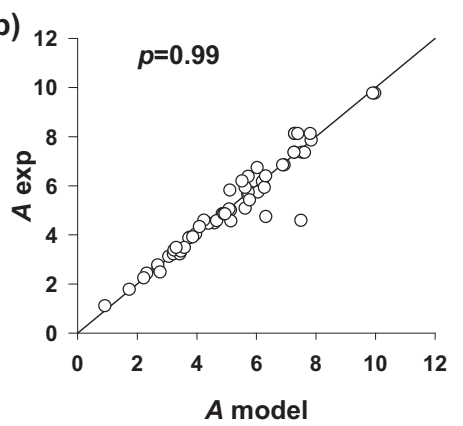

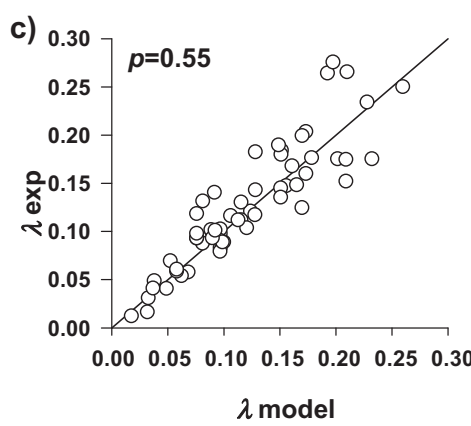

constant noise level
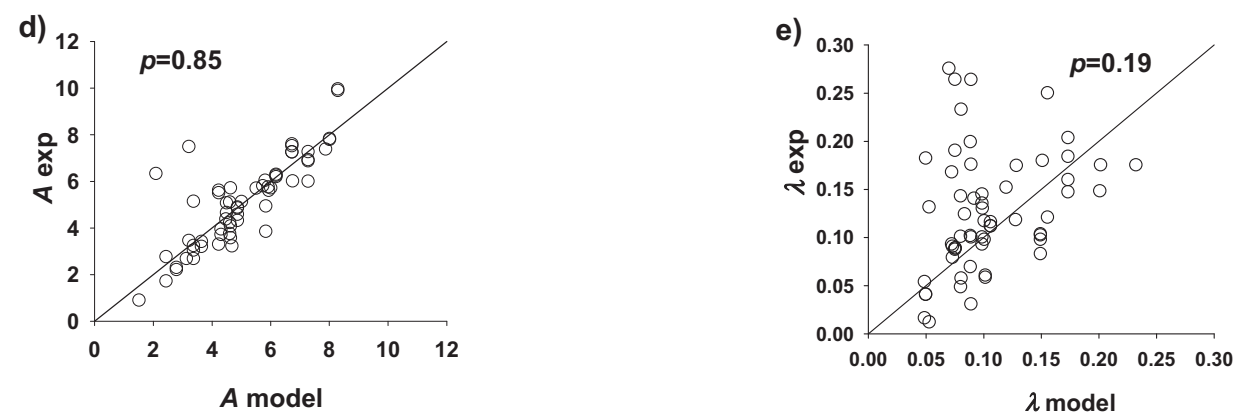

Fig. 4 The synaptic background noise and the number of oblique dendrites used to code different items can reproduce the experimental findings. a) Experimental data from 3 individuals tested with 12-word lists (black triangles), simulation findings (red circles) and the best fit of an exponential cumulative recall function for the simulation data (blue line); b) Values of A from the simulation vs those from the experiments; c) Values of $\lambda$ from the simulation vs those from the experiments; d) and e) comparison between experiments and simulations using a constant background noise. ( $p$ values refer to Mann-Withney rank sum test). 
Spera E. et al.: On the cellular mechanisms underlying working memory...

data, especially for $\lambda$ (Mann-Whitney rank sum test, $p=0.19$ ). These results suggest that the number of oblique dendrites used in a neuron to code for an item and the random background synaptic activity can be among the processes modulating individual WMC.
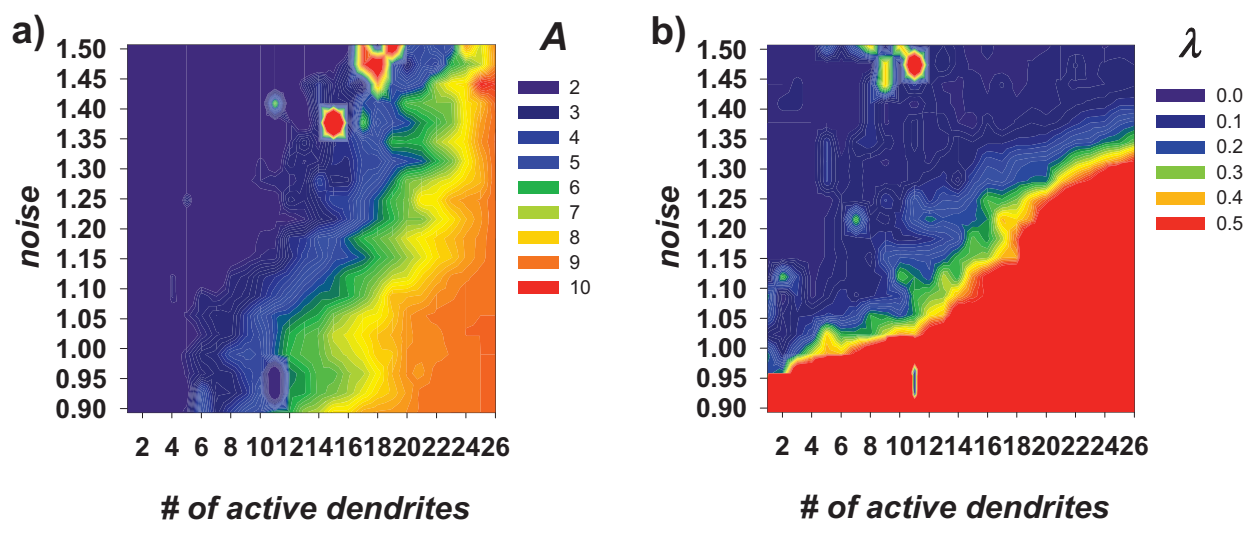

Fig. 5 The model is able to reproduce the full range of $\lambda$ and $A$ values observed experimentally. The contour plots show how a given combination of synaptic background noise and number of active dendrites coding for an item can modulate $A$ (a) and $\lambda(b)$ values in simulations of 9-word lists.

Using this approach, we were able to model the full range of $A$ and $\lambda$ values found experimentally (2-10, and $0-0.3$, respectively, see Fig. 1c). In Fig. 5 we show, for the case of 9-word lists, how the asymptotic number of items that could be recalled, $A$, and the rate of recall, $\lambda$, depend on the way in which a neuron represents an item (number of active dendrites) in the presence of a background synaptic noise. Analogous results were found for the cases of 6 - and 12-words lists (not shown). More specifically, we found that both $A$ and $\lambda$ tend to increase with the number of obliques used to recognize an item (Fig. 5, X-axis), and to decrease with the synaptic background noise (Fig. 5, Y-axis). These results suggest that individuals with different WMC performances, as measured by different values of $A$ and $\lambda$, can differ in the way they use to represent items at the single neuron level. Moreover it has to be noted that a value of zero for lamda means no spike was found.

The experimental data (see Fig. 1) show a considerable overlap between the cumulative recall function of individuals with high and low WMC. A typical example is shown in Fig. 6a for the case of 6 -word lists; $A$ and $\lambda$ values obtained from experiments with HWMC and LWMC individuals showed a substantial overlap and did not significantly differ from each other (Mann-Whitney rank sum test $p=0.488$ and $p=0.21$ for $A$ and $\lambda$, respectively).

This occurs because there are probably several different mechanisms that can more or less affect the recall process. This condition results in a very large variability in the experimental values that may confuse the interpretation of the ex- 

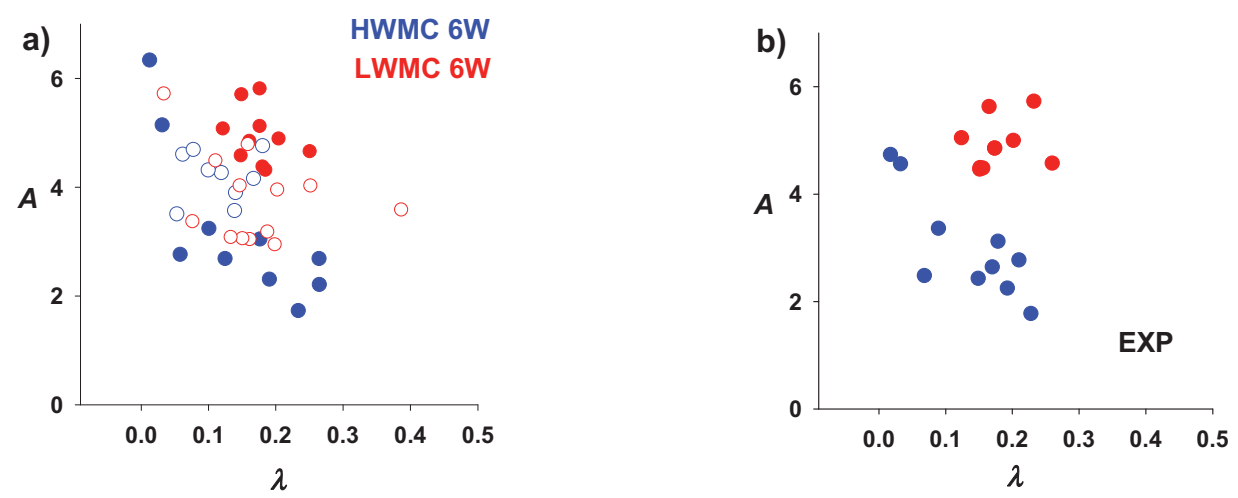

Fig. 6 Individuals with High WMC tend to use a higher number of oblique dendrites. a) Values for $A$ and $\lambda$ obtained from individuals tested with 6-word lists with a low (blue) or high (red) working memory capacity; solid circles represent the 10 best (red) and worst (blue) cases in terms of number of recalled words. the empty circles represent the worst cases among individuals with $H$-WMC (red) and the best case among individuals with $L$-WMC (blu). b) Values of $A$ and $\lambda$ from the simulations associated with the experimental 10 best (red) and worst (blue) cases.

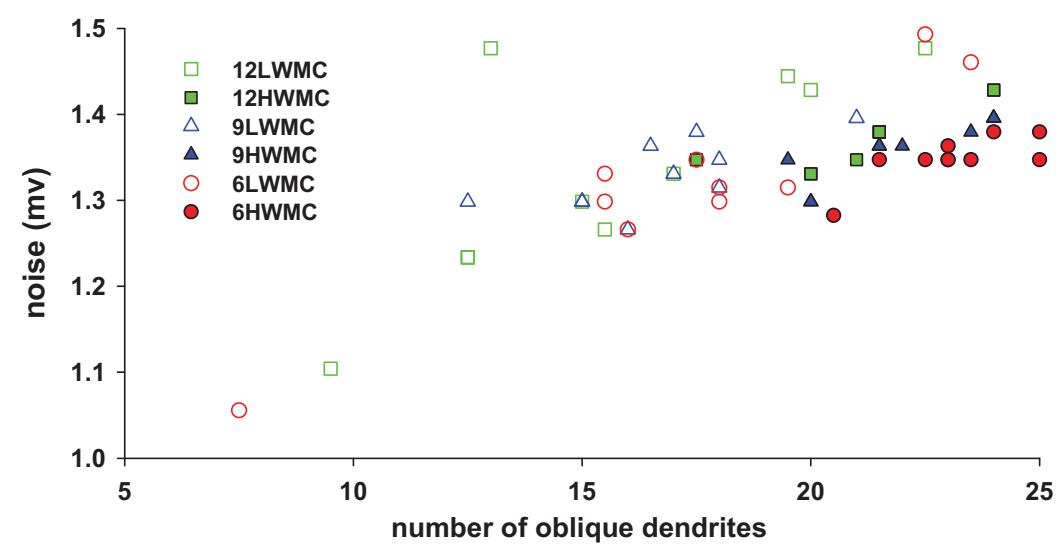

Fig. 7 Representation of the physiological parameter's space. Noise vs \# of active dendrites from simulations of the 10 best and worst cases for each experimental protocol (LWMC and HWMC individuals tested for 6-, 9-, and 12-word lists).

perimental findings. In the attempt to limit this effect, we restricted the analysis to the 10 best cases of HWMC and the worst 10 cases of LWMC (in terms of the number of recalled words). We assume that, in this way, we can indirectly isolate the effect of the most involved mechanisms. These cases are shown in Fig. 6a as closed symbols, and the relative values found from the simulations are shown in Fig. 6b. By carrying out the same analysis for the entire set of cases, we found that the values of $A$ obtained from HWMC and LWMC individuals were significantly 
Spera E. et al.: On the cellular mechanisms underlying working memory...

different under all conditions (i.e. 6, 9, and 12 words list, Mann-Whitney rank-sum test $p=0.017, p=0.002, p<0.001$, respectively), whereas the difference between $\lambda$ values was still not significant.

In Fig. 7 we present a summary of the model predictions for the 10 worst and best cases, in terms of the underlying physiological mechanisms (number of involved oblique dendrites and associated background noise) for each group of individuals tested under different conditions. The model suggests that there is a strong correlation between the number of oblique dendrites used to code an item and the level of background synaptic noise (Pearson's correlation coefficient $\rho=0.7$ ). Furthermore, as shown in Fig. 8, the model predicts that LWMC individuals use less oblique dendrites to represent an item, with respect to the HWMC individuals. Taken together, these results suggest that the different working memory capacity among humans can be explained, at least in part, by the specific way in which the hippocampal network of each individual has been wired, following (unknown) developmental, environmental, or natural conditions, to use more or less dendrites to encode and recall items.
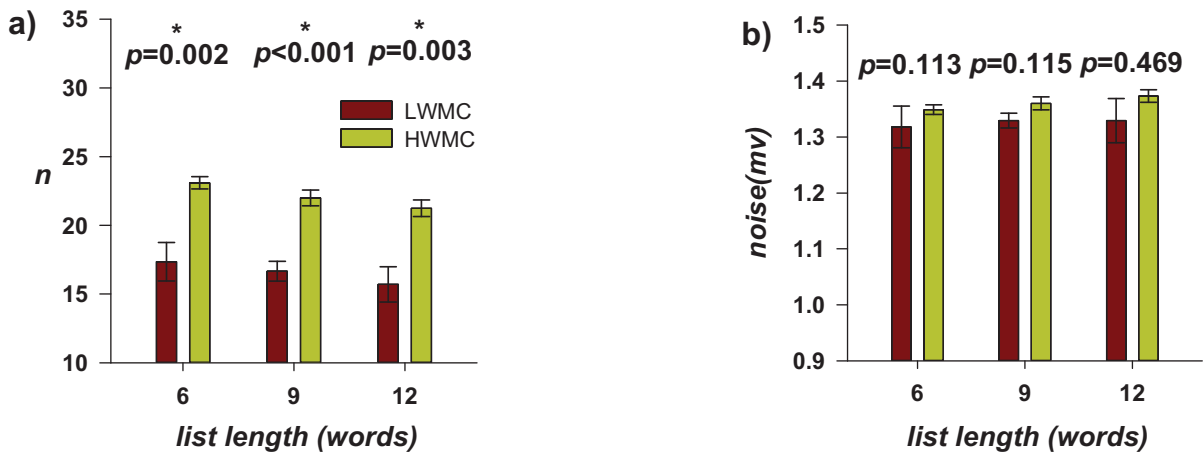

Fig. 8 The model predicts that $L W M C$ individuals use less oblique dendrites than $H W M C$ ones. a) Average number of active dendrites for simulations of the 10 best (brown) and worst (green) individuals, tested under lists of different length; in all cases, the difference in the average values was statistically significant (MannWhitney Signed Rank Sum test, $p$ indicated above bars). b) Average of noise intensity for simulations of the 10 best (brown) and worst (green) individuals, tested under lists of different length.

\section{Discussion}

In general, higher brain functions, such as working memory, are the end result of a number of independent mechanisms occurring at different levels, from brain region interactions to microscopic biochemical reactions. Here the focus was on those mechanisms occurring at the single cell level. From this point of view, our model suggests two main predictions: 1) the extent of the apical tree used to encode an item, and the synaptic background noise, may be responsible for the cumulative time of free recall observed in the experiments. The different memory capacity 
of humans can thus be qualitatively related to the basic mechanisms involved in dendritic signal integration; 2) HWMC individuals may use a greater number of oblique dendrites to encode words with respect to LWMC individuals. Assuming that any given individual encode an object using more than half of a neuron' oblique dendrites, the binomial coefficient is strictly decreasing. This implies that HWMC individuals will search for objects through a smaller set of possible combinations (see Methods), and supports the hypothesis [37] that the lower performance of LWMC individuals may be caused by a search through a larger set of items, which requires more computational time (assuming a random search through the possible objects). The conceptual framework we used in this work shares the idea of objects represented as a set of features (Nairne, 1990), but does not take into account the network dynamical reorganization and brain regions interaction that have been proposed to describe, in more general terms, a context-driven working memory system (O'Reilly and Frank, 2006). However, to the best of our knowledge, this is the first time that a link between psychological experiments and specific cellular mechanisms can be established. The results establish a proof of principle for the hypothesis that the extent of the apical tree used to encode an item and the synaptic background noise can play a major role in determining the overall number and rate at which items are recalled.

It should be stressed that in our approach we are not referring to any kind of grandmother cells. The fact that specific neurons explicitly code for specific objects is entirely dependent on the actual training session; this is consistent with the basic operations carried out by the hippocampus, which is universally considered as a working memory. Thus, the same neuron could very well be tuned through synaptic plasticity to code (or be involved in the coding of) any other object, according to whatever is presented in input during training. Our assumption of using a single neuron to code for an object should thus be considered as a simplified reduction of the sparse ensemble involved in the real system. It builds upon the clear experimental findings showing that firing of a single neuron can be a statistically significant indication of a specific input [17,31].

It may also be argued that a single realistic neuron is not all that different than a multilayer perceptron (MLP), which is proven to be able to approximate any function. Thus, in principle, we could have simply implemented a multi-layer artificial network to fit experimental data, a rather trivial process. However, the fact that a realistic neuron can be equivalent to a MLP (e.g. [29]) does not mean that a MLP built from scratch to fit experimental data can make experimentally testable predictions on the mechanisms at cellular level. As matter of fact, such a MLP would not (and cannot) give any clue on fundamental cellular processes such as dendritic signal integration. What can be eventually done is to first implement a biophysical model that takes into account the experimental data, and only then build an equivalent MLP based on it. This requires a biophysical model to be implemented first.

In principle, all psychological experiments analyzing the latency of delayed free recall using an exponential cumulative function can be interpreted in the same way as we have shown here. In spite of its simplicity, with our approach we were able to quantitatively take into account a number of experimental findings obtained under different conditions. A limitation of this approach is that it ignores the 
Spera E. et al.: On the cellular mechanisms underlying working memory...

many additional processes that must be involved in the physiological differentiation between HWMC and LWMC individuals. Taking into account these processes could also explain the considerable overlap between of the experimental curves obtained from High- and low-WMC individuals. However, this was outside the scope of this work, which focused on the possible main cellular mechanisms.

The reason why the extent of the apical tree used to encode an item and the synaptic background noise are able to modulate the cumulative time of free recall, can be understood by considering the special importance that hippocampal neurons have been shown to have for the recognition processes (e.g. [5]). We have previously shown [26] how synaptic inputs, eliciting local dendritic spikes on a specific combination of oblique dendrites of hippocampal CA1 pyramidal neurons, can be one of the mechanisms leading to objects recognition. The random synaptic background noise can have a quite strong impact on this process, considering that it can be related to the internal activity elicited by evoked sensory responses [8]. Too low or too high levels of noise act in opposite ways to modulate the number of combinations of active dendrites able to generate a somatic action potential (and thus a recognition signal): low levels will make it easier for a neuron to fire an AP, whereas high levels shunt too many combinations and result in a lower spike probability. In the context investigated here, these effects can result in an object (e.g. a specific word from a list) being recalled sooner or later during the free recall process.

The number of active dendrites used to encode objects (a list of words, in the experiments discussed in this work) implies a different number of possible ways in which the activity in a group of dendrites can be integrated in such a way to generate a somatic action potential. For example, the particular CA1 pyramidal neuron morphology we used for all simulations has 26 oblique dendrites. If objects are coded using 17 obliques (for example corresponding to 17 different features), this particular neuron can be involved in the encoding of up to $\left(\begin{array}{l}26 \\ 17\end{array}\right)=3,124,550$ different objects. Using 22 obliques, this number would be reduced to $\left(\begin{array}{l}26 \\ 22\end{array}\right)=$ 14,950 .

Our model predicts that HWMC individuals may use a greater number of oblique dendrites to encode words with respect to LWMC individuals. Assuming that any given individual encode an object using more than half of a neuron' oblique dendrites, the binomial coefficient is strictly decreasing. This implies that HWMC individuals will search for objects through a smaller set of possible combinations, and supports the hypothesis [37] that the lower performance of LWMC individuals may be caused by a search through a larger set of items, which requires more computational time (assuming a random search through the possible objects). From a more general point of view, our study predicts that the differences between High and Low WMC individuals could be in part explained with the same mechanism for any mammal, assuming that the search strategy is the same. Of course this does not also imply that working memory tasks are carried out in the same way. Higher brain functions require circuits and networks operations (which were outside the scope of this work) with a role that cannot be overemphasized.

Two important next steps can be foreseen for this model: 1) a study of how alterations in the balance between excitatory and inhibitory pathways can affect signal integration, and thus memory capacity; this is an important mechanism that 
we have not investigated in this work; although most alterations lead to network malfunctioning (reviewed in [10]), it could be speculated that moderate or transient deviations can instead significantly affect the ability to encode and recall items; 2) an investigation of the memory capacity degradation with the progression of brain pathologies such as Alzheimer's disease and the possible ways to ameliorate this condition $[3,6]$.

The overall picture emerging from this work is thus one in which individuals with high WMC have implemented a strategy that, during the encoding phase, allows them to store as many as possible features of the objects they are being shown. During recall, this results in a search through a smaller set of possible objects. The model suggests that, in order for this strategy to be properly organized, HWMC individuals generate an internal dynamics that results in a higher synaptic background activity from within the hippocampus itself or from other brain regions.

\section{Acknowledgement}

This work was supported by the "Piattaforma regionale di ricerca traslazione per la salute" - P.O. FESR 2007/2013 - linea di intervento 4.1.2.A, under Grant CUPB65E12000570008. We thank the CINECA Consortium (Bologna, Italy) for granting access to their IBM BlueGene/Q supercomputer system.

\section{References}

[1] ANDERSON P., MORRIS R., AMARAL D., BLISS T., O'KEEFE J. Historical perspective: proposed functions, biological characteristics, and neurobiological models of the hippocampus. The Hippocampus Book. New York: Oxford University Press. 2006, doi: 10.1093/acprof : oso/9780195100273.003.0002.

[2] ASCOLI G.A. Mobilizing the base of neuroscience data: the case of neuronal morphologies. Nat. Rev. Neurosci. 2006, 7, pp. 318-24, doi: 10.1038/nrn1885.

[3] BiAnChi D., DE Michele P., MARChetTi C., TiRozzi B., CUOMO S., MARIE H., MICHELE M. Effects of increasing CREB-dependent transcription on storage and recall processes in a hippocampal CA1 microcircuit. Hippocampus. 2014, doi: 10.1002/hipo. 22212.

[4] BREWIN C.R., BEATON A. Thought suppression, intelligence, and working memory capacity. Behaviour Research and Therapy. Elsevier Press. 2002, 40, pp. 923-930, doi: 10.1016/ S0005-7967(01)00127-9.

[5] CERF M., ThiRUVengadam N., MORMann F., KRASKOV A., QUiRoGa R.Q., KOCH C., FRIED I. On-line, voluntary control of human temporal lobe neurons. Nature 467. 2010, 467, pp. 1104-1108, 2010. doi: 10.1038/nature09510.

[6] CULMONE V., MIGLIORE M.Progressive effect of beta amyloid peptides accumulation on CA1 pyramidal neurons: a model study suggesting possible treatments. Front Comput Neurosci. 2012, 6, doi: 10.3389/fncom.2012.00052.

[7] DANEMAN M., CARPENTER P.A. Individual differences in working memory and reading. Journal of Verbal Learning and Verbal Behavior. 1980, 19, pp. 450-466, doi: 10.1016/ S0022-5371(80) 90312-6.

[8] DESTEXHE A. Intracellular and computational evidence for a dominant role of internal network activity in cortical computations. Curr Opin Neurobiol. 2011, 21, pp. 717-725, doi: $10.1016 / j$. conb. 2011.06.002.

[9] DeSTeXHE A. RUDOLPH M., FELLOUS J.M., SEJNOWSKI T.J. Fluctuating synaptic conductances recreate in vivo-like activity in neocortical neurons. Neuroscience. 2001, 107, pp. 13-24. doi: $10.1016 / j$.conb.2011.06.002. 
Spera E. et al.: On the cellular mechanisms underlying working memory...

[10] EICHLER S.A., MEIER J.C. E-I balance and human diseases - from molecules to networking. Front Mol Neurosci. 2008, 1(2), doi: 10.3389/neuro.02.002.2008.

[11] ENGLE R.W., KANE M.J. Executive attention, working memory capacity, and a two-factor theory of cognitive control. The psychology of learning and motivation. New York: Elsevier. 2004, 44, pp. 145-199, doi: 10.1016/S0079-7421(03) 44005-X.

[12] ENGLE R.W., KANE M.J., TUHOLSKI S.W. Individual differences in working memory capacity and what they tell us about controlled attention, general fluid intelligence, and functions of the prefrontal cortex. Models Of Working Memory: Mechanisms of Active Maintenance and Executive Control. Cambridge University Press. 1999, pp. 102-134, edited by Akira Miyake And Priti Shah.

[13] GASPARINI S., LOSONCZY A., CHEN X., JOHNSTON D., MAGEE J.C. Associative pairing enhances action potential back-propagation in radial oblique branches of CA1 pyramidal neurons. J. Physiol. 2007, 580(3), pp. 787-800, doi: 10.1113/jphysiol.2006.121343.

[14] GASPARINI S., MIGLIORE M., MAGEE J.C. 2004. On the initiation and propagation of dendritic spikes in CA1 pyramidal neurons. The Journal of Neuroscience 24. 2004, pp. 11046-11056, doi: 10.1523/JNEUROSCI. 2520-04.

[15] HINES M.L., CARNEVALE N.T. The NEURON simulation environment. Neural Comput . 1997, 9(6), pp. 1179-1209, doi: 10.1162/neco.1997.9.6.1179.

[16] ISHIZUKA N., COWAN W.M., AMARAL D.G. A quantitative analysis of the dendritic organization of pyramidal cells in the rat hippocampus. J. Comp. Neurol. 1997, 362(1), pp. 17-45, doi: 10.1002/cne.903620103.

[17] ISON M.J., MORMANN F., CERF M., KOCH C., FRIED I., QUIROGA R.Q. Selectivity of pyramidal cells and interneurons in the human medial temporal lobe. J. Neurophysiol. 2011, 106(4), pp 1713-21, doi: 10.1152/jn.00576.2010.

[18] JOHNSTON D., AMARAL D.G. Hippocampus. The Synaptic Organization of the Brain. New York: OxfordUniversity Press. 2004, doi: 10.1093/acprof : oso/9780195159561.003. 0011.

[19] KANE M.J., ENGLE R.W. Working memory capacity, proactive interference, and divided attention: Limits on long-term retrieval. Journal of Experimental Psychology: Learning, Memory, and Cognition. 2000, 26, pp. 333-358, doi: 10.1037/0278-7393.26.2.336.

[20] KATZ Y., MENON V., NICHOLSON D.A., GEINISMAN Y., KATZ W.L., SPRUSTON N. Synapse distribution suggests a two-stage model of dendritic integration in CA1 pyramidal neurons. Neuron. 2009, 63(2), pp. 171-177, doi: 10.1016/j.neuron.2009.06.023.

[21] LOSONCZY A., MAGEE J.C. Integrative properties of radial oblique dendrites in hippocampal CA1 pyramidal neurons. Neuron. 2006, 50, pp. 291-307, doi: 10.1016/j.neuron. 2006 . 03.016.

[22] MAKARA J.K., LOSONCZY A., WEN Q., MAGEE J.C. Experience-dependent compartmentalized dendritic plasticity in rat hippocampal CA1 pyramidal neurons. Nat Neurosci. 2009, 12(12), pp. 1485-7, doi: 10.1038/nn.2428.

[23] MEGIAS M., EMRI Z., FREUND T.F., GULVAS A.I. Total number and distribution of inhibitory and excitatory synapses on hippocampal CA1 pyramidal cells. Neuroscience. 2001, 102, pp. 527-540, doi: 10.1016/S0306-4522(00)00496-6.

[24] MIGLIORE M. On the integration of subthreshold inputs from Perforant Path and Schaffer Collaterals in hippocampal CA1 pyramidal neurons. J. Comput Neurosci. 2003, 14(2), pp. 185-192, doi: $10.1023 / \mathrm{A}: 1021906818333$.

[25] MIGLIORE M., FERRANTE M., ASCOLI G.A. Signal propagation in oblique dendrites of CA1 pyramidal cells. J. Neurophysiol. 2005, 94, pp. 4145-4155, doi: 10.1152/jn.00521.2005.

[26] Migliore M., NOVARA G., TEGOLO D. Single neuron binding properties and the magical number 7. Hippocampus. 2008, 18, pp. 1122-1130, doi: 10.1002/hipo. 20480.

[27] MIGLIORE M., SHEPHERD G.M. Emerging rules for the distributions of active dendritic conductances. Nature Rev. Neurosci. 2002, 3, pp. 362-370, doi: 10.1038/nrn810.

[28] MORRIS R. Theories of hippocampal function. The Hippocampus Book. New York: Oxford University Press. 2006, doi: 10.1093/acprof : oso/9780195100273.003.0013. 


\section{Neural Network World 4/2016, 335-350}

[29] POIRAZI P., BRANNON T., MEL B.W. Pyramidal neuron as two-layer neural network. Neuron. 2003, 37, pp. 989-999, doi: 10.1016/S0896-6273(03)00149-1.

[30] QUIROGA R.Q., MUKAMEL R., ISHAM E.A., MALACH R., FRIED I. Human singleneuron responses at the threshold of conscious recognition. Proc Natl Acad Sci USA. 2008, 105(9), pp. 3599-3604, doi: 10.1073/pnas.0707043105.

[31] QUIROGA R.Q., REDDY L., KREIMAN G., KOCH C., FRIED I. Invariant visual representation by single neurons in the human brain. Nature. 2005, 435, 1102-1107, doi: 10.1038/ nature03687.

[32] SEGMILLER, JANELLE K., WATSON, JASON M., STRAYER, DAVID L. Individual differences in susceptibility to inattentional blindness. Journal of Experimental Psychology: Learning, Memory, and Cognition. 2011, 37(3), pp. 785-791, doi: 10.1037/a0022474.

[33] SQUIRE L.R., STARK C.E.L., CLARK R.E. The medial temporal lobe. Ann. Rev. Neurosci. 2004, 27, pp. 279--306, doi: 10.1146/annurev . neuro.27.070203.144130.

[34] TURNER M.L., ENGLE R.W. Is working memory capacity task dependent? Journal of Memory and Language. 1989, 28, pp. 127-154, doi: 10.1016/0749-596X (89) 90040-5.

[35] UHLENBECK G.E., ORNSTEIN L.S. On the theory of the Brownian motion. Phys. Rev. 1930, 36, pp. 823-841, doi: 10.1103/PhysRev.36.823.

[36] UNSWORTH N. Individual differences in working memory capacity and episodic retrieval: examining the dynamics of delayed and continuous distractor free recall. Journal of Experimental Psychology: Learning, Memory, and Cognition. 2007, 33(6), pp. 1020-1034, doi: $10.1037 / 0278-7393.33 .6 .1020$

[37] UNSWORTH N., ENGLE R.W. The nature of individual differences in working memory capacity: Active maintenance in primary memory and controlled search from secondary memory. Psychological Review. 2007, 114, pp. 104-132, doi: 10.1037/0033-295X.114.1.104.

[38] WILHELM O., HILDEBRANDT A., OBERAUER K. What is working memory capacity, and how can we measure it? Frontiers in Psychology. 2013, pp. 4-433, doi: 10.3389/fpsyg. 2013.00433.

[39] WIXTED J.T., ROHRER D. Analyzing the dynamics of free recall: An integrative review of empirical literature. Psychonomic Bulletin and Review. 1994, 1, pp. 89-106, doi: 10.3758/ BF03200763. 